\title{
Comparison of Effectiveness of Fennel versus Vitamin (E) on Primary Dysmenorrhea among Benha University Nursing Students
}

\author{
Al-Shaimaa A. Mohamed ${ }^{1}$, Galal A. Elkholey², Soad A. Ramadan ${ }^{3}$, Eman M. \\ Abd El-hakam ${ }^{4}$ \\ ${ }^{1}$ Nursing Specialist and Teacher at Secondary Technical Nursing School in Qalyube, ${ }^{2}$ Professor of \\ Obstetrics \& gynecology, Faculty of Medicine, Benha University, ${ }^{3}$ Assistant Professor of Obstetrics \\ and Woman Health Nursing, Faculty of Nursing, Benha University, ${ }^{4}$ Lecturer of Obstetrics and \\ Woman Health Nursing Faculty of Nursing, Benha University
}

\begin{abstract}
Dysmenorrhea is pelvic pain usually, associated with menstrual periods. The purpose of the current study is to study the effect of fennel on relieving primary dysmenorrhea compared to vitamin E. Design: A quasi-experimental design was used. Setting: Faculty of Nursing, Benha University. Sampling: A Purposive sample included 116 students divided into two equal groups (58 fennel and 58 vitamin E groups). Instruments: 1- A structured interviewing questionnaire sheet consisted of four parts. 2- Visual Analog Scale. Results: One month before fennel and vitamin E interventions, female nursing students who had severe menstrual pain, lumbo-sacral backache, pain radiating down anterior thigh and breast tenderness were $34.0 \%$ \& $38.6 \%, 84.9 \%$ \& $91.2 \%, 54.7 \%$ $\& 54.4,73.7 \% \& 24.6 \%$. Besides at $-3^{\text {rd }}$ month follow-up, female nursing students who had no (menstrual pain, lumbo-sacral backache, pain radiating down anterior thigh and breast tenderness) were $41.5 \% \& 19.3 \%, 50.9 \% \& 28.1 \%, 77.4 \% \& 61.4 \%, 94.3 \% \& 87.7 \%$ respectively. Conclusion: Fennel tea and vitamin E had strong positive effect on dysmenorrhea relief. But students who received fennel seed's tea experienced less severe dysmenorrhea than students who received vitamin E. Recommendation: nurse should encourage females with dysmenorrhea to try Fennel tea or vitamin $\mathrm{E}$ for four subsequent menstrual cycles to ensure their efficacy on dysmenorrhea relief (starting from two days before menstrual bleeding onset to three days after menstrual bleeding onset).
\end{abstract}

Keywords: Dysmenorrhea; Fennel tea; Nursing students; Vitamin E.

\section{INTRODUCTION}

Dysmenorrhea is painful menstrual cramps of uterine origin; It occurs before or soon after the onset of menses and is spasmodic in nature, with discomfort in the lower abdomen which may radiate to the lower back or down the legs. Some women also have diarrhea, nausea, and vomiting. It is most common in young women who have not been pregnant (Leifer, 2015). Dysmenorrhea may be primary or secondary.

Primary dysmenorrhea is characterized by the absence of any specific pelvic pathologic condition; Secondary dysmenorrhea has underlying pathology such as anatomic abnormality, or infection such as pelvic inflammatory disease. The most common cause of secondary dysmenorrhea in adolescents is endometriosis (Sucato and Burstein, 2016).

Also in Egypt, there was a study tested the epidemiology of dysmenorrhea among workers in Upper Egypt, the study reported higher rates of dysmenorrhea during the past 12 months (94.6\%) amongst the working women, compared to their controls $(90.7 \%) \quad(\mathrm{P}<0.05)$ (Arafa, et al., 2018).

Primary dysmenorrhea can be treated by non-steroidal anti-inflammatory agents, oral contraceptives hormonal drugs...etc. and non-pharmacological options e.g. exercise local heat, behavioral interventions, and dietary supplements as all herbs and vitamins (Burnett and Lemyre, 2017). 


\section{Comparison of Effectiveness of Fennel versus Vitamin (E) on Primary Dysmenorrhea among Benha University Nursing Students}

One of these herbal remedies is fennel which could be effective on relieving dysmenorrhea (Ghodsi and Asltoghiri, 2014). Also there are different types of vitamins which were used in the management of dysmenorrhea (Kauphal, 2018) such as vitamin $E$ which has positive effect on primary dysmenorrhea (Akhlaghi et al., 2009).

\section{PURPOSE}

The purpose was to compare the effect of fennel versus vitamin $\mathrm{E}$ on relieving primary dysmenorrhea.

\section{HYPOTHESIS}

The students who receive fennel seeds' tea will experience less severe dysmenorrhea compared to the students who will receive vitamin $\mathrm{E}$.

\section{METHODS}

Research design:- A quasi-experimental design (two parallel groups).

Research Settings:- Faculty of Nursing, Benha University.

Sampling:- A sample of 116 nursing students from first academic year, were chosen after pre-assessment screening phase and compatible with the inclusion criteria. the selected students were randomly divided into two equal groups: Fennel seed' tea group (1) $=58$ students. Vitamin $\mathbf{E}$ group (2) $=58$ students.

\section{Inclusion Criteria:}

1. Nursing student.

2. Suffering from primary dysmenorrhea.

3. At least History of one year regular menses.

4. Age range from 17 th years old to 18 th years old.

5. Qualiobeya governorate inhabitants.

\section{Exclusion Criteria:}

1. Married.

2. Suffering from medical diseases.as epilepsy, DM, Hypertension, heart disease...etc.
3. Receiving any medications or contraceptives, herbal or complementary remedies.

4. Following special dietary regiment.

5. Suffering from allergy to fennel, vitamin $\mathbf{E}$ or any type of food.

\section{Instruments:}

Instrument one: A Structured Interviewing Questionnaire Sheet: it is designed in a simple Arabic language by the researcher after review of literatures related to dysmenorrhea subject (Abd El-Hameed, 2011; Kannan, et al., 2015; Shehata, 2015; Wetwet, 2016; Abo Elnor, 2017) and consisted of four parts:-

* Part one: Social Data Sheet: it contained questions about name, telephone number, age, residence, anthropometric measurements (as weight, height and BMI), socio-economic status ...etc.

* Part two: Menstrual History Sheet :It included questions about menarche, duration and interval of menstruation, menstrual information and sources of information.

* Part three: Menstrual Pain Pattern Sheet: It Composed of questions about severity, duration of menstrual pain, its effect on daily activity, learning capacity and scholar abscentism, bleeding (amount of pads/day and nature of bleeding).

* Part four: Menstrual Associated Symptoms Sheet: it included questions about associated symptoms as lumbosacral backache, pain radiating down anterior thigh, breast tenderness, nausea, vomiting, diarrhea, headache, dizziness and fainting.

Scoring system for pain:

\begin{tabular}{|l|c|}
\hline Scoring Items & Score \\
\hline No pain & (Zero) \\
\hline Mild pain & $(2.5)$ \\
\hline Moderate pain & $(5)$ \\
\hline Severe pain & $(7.5)$ \\
\hline Intolerable pain & $(10)$ \\
\hline
\end{tabular}


Scoring system for bleeding:

\begin{tabular}{|l|c|}
\hline Scoring Items & Score \\
\hline Mild bleeding & ( $\leq 2$ pads/day $)$ \\
\hline Moderate bleeding & (3-5 pads/day) \\
\hline Severe bleeding & ( $\geq 6$ pads/day) \\
\hline
\end{tabular}

Scoring system for lumbo-sacral backache, pain radiating down anterior thigh, breast tenderness, nausea, vomiting, diarrhea, headache, dizziness and fainting:

\begin{tabular}{|l|c|}
\hline Scoring Items & Score \\
\hline Yes & 1 \\
\hline No & Zero \\
\hline
\end{tabular}

\section{Reliability:}

The reliability of the structured interview questionnaire sheet was measured by Crombach's alpha and the value was (0.71).

Instrument two: Visual Analog Scale (VAS):It is a measurement tool to measure the intensity of pain. This scale was adopted from (Sriwatanakul, et al., 1983 and Gruenberg, et al., 1996) that consisted of a blank $10 \mathrm{~cm}$ line anchored at each end by adjectives that describe the extremes of pain for ease of measurement.

\section{Scoring system for VAS:}

\begin{tabular}{|l|c|}
\hline Item & Score \\
\hline No pain & (Zero) \\
\hline Mild pain & $(2.5)$ \\
\hline Moderate pain & $(5)$ \\
\hline Severe pain & $(7.5)$ \\
\hline Worst possible pain & $(10)$ \\
\hline
\end{tabular}

\section{Reliability:}

The reliability of visual analog scale was measured by Crombach's alpha and the value was (0.74).

\section{Validity:}

The developed instruments were reviewed for content, appropriateness of items and measuring concepts by a jury panels composed of 5 experts (three professors in maternity and two professors in obstetrics), Necessary modifications were done according to panel judgment.

\section{Procedure:}

1) An official permission was submitted to the dean of the Faculty of Nursing at Benha University explaining the aim of the study and time of data collection, The study was carried out from the beginning of (February 15, 2017 to the end of July 2017). It was done on two days per week from $10 \mathrm{am}$. to $1 \mathrm{Pm}$. (Saturday and Sunday).

2) The researcher conducted pre-assessment screening phase which was simple questionnaire to screen for students who are compatible with the inclusion criteria. Included the following data: name, academic year, regularity of menstruation, presence of dysmenorrhea, marital status, medical history (diseases, medications, using (contraceptives, herbal or complementary) remedies), residence and allergy.

3) After revising the screening sheet, the numbers of students compatible with the inclusion criteria were one hundred twenty nine students (129) and listed.

4) Instructions were given to students to fill a structured interviewing questionnaire sheet.

5) The researcher interviewed students individually; written informed consent was taken, till the sample size was completed, time taken to complete this sheet 30 minutes.

6) The students were also instructed to determine the severity of dysmenorrhea by using Visual Analog Scale before research application.

7) The studied sample was divided randomly into two equal groups by a simple random sample:

Fennel Seeds' Tea Group1: (58) female students who applied nursing intervention with fennel seeds` tea to relieve dysmenorrhea and they were directed to use a daily cup of warm 


\section{Comparison of Effectiveness of Fennel versus Vitamin (E) on Primary Dysmenorrhea among Benha University Nursing Students}

infusion of fennel seeds' tea (1-1 $1 / 2$ teaspoons of seeds per day as a typical dose) (Geller and Simone, 2011) before $48 \mathrm{hrs}$. before their menses till three days after the beginning of menstruation and they were given Fennel Seeds' tea Leaflet as guidance.

* Vitamin E Group2: (58) female students used vitamin $\mathrm{E}$ to relieve dysmenorrhea and they were instructed to use (400 IU) as a single capsule after dinner 48 hrs. before their menstruation till three days after the beginning of menstruation. This dose was determined by asking the obstetrician physician and literature reviewing (Kauphal, 2018) and they were provided vitamin $\mathrm{E}$ leaflet as guidance.

* The studied sample was directed to apply the intervention for four subsequent months (month for research application and three months for follow-up), they were asked to fulfill menstrual characteristics(menstrual history and pain pattern), menstrual associated symptoms sheets and sign in the Visual Analog Scale (VAS) monthly for observation and follow-up purposes.

\section{Pilot study:}

A pilot study was conducted on 13 female nursing students (10\% of the sample); it was done to check the clarity and feasibility of the instruments. Necessary modifications was done in the form of adding or omission of some questions and change types of some questions from open ended questions to closed ended questions; pilot study was excluded from the sample.

\section{Ethical considerations:}

Ethical aspects included the following 1A written informed consent for sharing in the study and explanation of the purpose student after explanation of the aim of the study to gain their confidence with the study. 2-Participants should be reassured that data collected will be treated confidentiality and will be used only in the research. 3- No harm for participants. 4- Each participant had the right to withdraw from participating at any time.

\section{DATA ANALYSIS}

Researcher verified data prior to a computerized entry. The Statistical Package for Social Sciences (SPSS version 20) was used for that purpose, followed by data analysis and tabulation. Descriptive statistics were applied: As Mean, standard deviation and test for quantitative data, Also frequency, FET and X2 distribution used for qualitative data. Statistical significance difference was considered if $\mathrm{p} \leq 0.05$ and a highly significant level value was considered when $\mathrm{p} \leq 0.001$.

\section{RESULTS}

Table 1 demonstrates distribution of the studied sample related to social characteristics that rural area residents were $77.4 \%$ and $84.2 \%$ in group1 (fennel) and group 2(vitamin E). Besides university graduate fathers were $43.4 \%$ and $49.1 \%$ in group $1 \& 2$ respectively, Also working fathers were $100 \%$ and 94.7\% respectively; In addition, housewives were $71.7 \%$ and $59.6 \%$ respectively, and normal BMI was $69.8 \%$ and $73.7 \%$ respectively. There was no statistical significant difference between two groups regarding to social data, $(\mathrm{P}>0.05)$.

Table 2 shows distribution of the studied sample according to menstrual history before intervention that age at menarche between approximately two thirds of the sample was $67.9 \%$ and $66.7 \%$ ranged from 12 to $15 \mathrm{yrs}$. in group $1 \& 2$. Also average of menstrual duration in group $1 \& 2$ was 3-8 and 3-12 days respectively, more than half of them obtained 


\section{Comparison of Effectiveness of Fennel versus Vitamin (E) on Primary Dysmenorrhea among Benha University Nursing Students}

information about menses from their mothers $(54.7 \%$ and $56.1 \%$ in group $1 \& 2)$. There was no statistical significant difference between two groups regarding to menstrual history, $(\mathrm{P}>0.05)$.

Table 3 illustrates distribution of the studied sample related to menstrual characteristics that more than half of them have moderate to severe pain in group 1 (50.9\% and $34.0 \%)$ and group 2 (38.6\% and $38.6 \%$ ). Average dysmenorrheal pain duration was 2 to 5 days in two groups. More than three quarters of sample didn't have information about fennel in group 1 $(75.5 \%)$ and group $2(78.9 \%)$ and vitamin E (86.8\% and $80.7 \%)$. There was no statistical significant difference between two groups regarding menstrual characteristics, $(\mathrm{P}>0.05)$.

Table 4 reveals distribution of the studied sample according to menstrual associated symptoms before intervention that more than three quarters of the sample have lumbo-sacral backache in group 1 $(84.9 \%)$ and group $2(91.4 \%)$. Range of visual analog scale score is 3-10 and 210. There was no statistical significant difference between two groups regarding menstrual associated symptoms, $(\mathrm{P}>0.05)$.

Table 5 shows distribution of the studied sample related to menstrual characteristics one month after intervention that mild and moderate menstrual pain accompanied was $41.5 \%$ and $35.8 \%$ for group 1 and was $35.1 \%$ and 42.1 for group2. there was a statistical significant difference between two groups observed in mean menstrual pain duration (days) which was $2.06 \pm 0.59$ and $1.78 \pm 0.63$ days respectively, $(\mathrm{P}<0.05)$.

Table 6 shows distribution of the studied sample according to menstrual associated symptoms one month after intervention that severe and moderate lumbosacral backache were available between $41.5 \%$ and $18.9 \%$ in group 1 and were $34.9 \%$ and $21.1 \%$ in group2, while severe and moderate pain radiating down anterior thigh were $24.5 \%$ and $17.0 \%$ in group 1 and were $26.3 \%$ and $19.3 \%$ in group2. There was no statistical significant difference between two groups regarding menstrual associated symptoms, $(\mathrm{P}>0.05)$.

Table 7 demonstrates distribution of the studied sample related to menstrual characteristics at 3rd month follow-up that menstrual duration was 3-7 days for group1\&2. Also menstrual interval was 21-30 and 21-28 days for group1\&2. there were highly statistical significant differences of moderate menstrual pain was $1.9 \%$ and $35.1 \%$ in group $1 \& 2$ respectively, also no menstrual pain effect on daily activity was $83.0 \%$ and $56.1 \%$ in group $1 \& 2$ respectively, $(\mathrm{p}<0.01)$. Beside that there were statistical significant differences as one day absence related to menses was $32.1 \%$ and $54.4 \%$ and moderate amount of menstrual bleeding (3-5 pads/day) was $26.4 \%$ and $45.6 \%$ in group1\&2 respectively, $(\mathrm{P}<0.05)$.

Table 8 reveals distribution of the studied group according to menstrual associated symptoms at 3rd month follow-up that there were statistical significant differences as the students who had no lumbo-sacral backache $50.9 \%$ and $28.1 \%$ in group1\&2, then nausea was $7.5 \%$ and $21.1 \%$ in group $1 \& 2$ respectively, In addition, vomiting was $5.7 \%$ and $21.1 \%$ in group1\&2 respectively, besides diarrhea was $5.7 \%$ and $19.3 \%$ in group $1 \& 2$ respectively, after that headache was $11.3 \%$ and $31.6 \%$ in group $1 \& 2$ respectively, and dizziness was $17.0 \%$ and $38.6 \%$ in group1\&2respectively, ( $\mathrm{P}<0.05)$.

Table (1): Distribution of the studied sample related to social characteristics:

$(\mathrm{N}=110)$. 

among Benha University Nursing Students

\begin{tabular}{|c|c|c|c|c|c|c|c|c|}
\hline \multirow[t]{2}{*}{ Social Characteristics } & \multicolumn{2}{|c|}{$\begin{array}{c}\text { Fennel Group } 1 \\
\quad(\mathrm{~N}=53)\end{array}$} & \multicolumn{2}{|c|}{$\begin{array}{l}\text { Vitamin E } \\
\text { Group } 2 \\
(\mathrm{~N}=57)\end{array}$} & \multicolumn{2}{|c|}{ Total } & \multirow[t]{2}{*}{ FET } & \multirow[t]{2}{*}{ P-Value } \\
\hline & No. & $\%$. & No. & $\%$ & No. & $\%$ & & \\
\hline $\begin{array}{l}\text { Residence. } \\
\text { Rural } \\
\text { Urban }\end{array}$ & $\begin{array}{l}41 \\
12\end{array}$ & $\begin{array}{l}77.4 \\
22.6\end{array}$ & $\begin{array}{c}48 \\
9\end{array}$ & $\begin{array}{l}84.2 \\
15.8\end{array}$ & $\begin{array}{l}89 \\
21\end{array}$ & $\begin{array}{l}80.9 \\
19.1\end{array}$ & $\mathrm{X}_{2}=0.84$ & $0.36^{\mathrm{ns}}$ \\
\hline $\begin{array}{l}\text { Educational level of } \\
\text { students' mother. } \\
\text { Illiterate } \\
\text { Read and write } \\
\text { Primary } \\
\text { Secondary } \\
\text { University } \\
\end{array}$ & $\begin{array}{c}4 \\
0 \\
6 \\
31 \\
12 \\
\end{array}$ & $\begin{array}{c}7.5 \\
0.0 \\
11.3 \\
58.5 \\
22.6 \\
\end{array}$ & $\begin{array}{c}3 \\
3 \\
7 \\
32 \\
12\end{array}$ & $\begin{array}{c}5.3 \\
5.3 \\
12.3 \\
56.1 \\
21.1 \\
\end{array}$ & $\begin{array}{c}7 \\
3 \\
13 \\
63 \\
24\end{array}$ & $\begin{array}{c}6.4 \\
2.7 \\
11.8 \\
57.3 \\
21.8 \\
\end{array}$ & 2.82 & $0.62^{\mathrm{ns}}$ \\
\hline $\begin{array}{l}\text { Educational level of } \\
\text { student's father. } \\
\text { Illiterate } \\
\text { Read and write } \\
\text { Primary } \\
\text { Secondary } \\
\text { University } \\
\end{array}$ & $\begin{array}{c}1 \\
3 \\
5 \\
21 \\
23 \\
\end{array}$ & $\begin{array}{c}1.9 \\
5.7 \\
9.4 \\
39.6 \\
43.4\end{array}$ & $\begin{array}{c}3 \\
0 \\
3 \\
23 \\
28 \\
\end{array}$ & $\begin{array}{c}5.3 \\
0.0 \\
5.3 \\
40.4 \\
49.1 \\
\end{array}$ & $\begin{array}{c}4 \\
3 \\
8 \\
44 \\
51\end{array}$ & $\begin{array}{c}3.6 \\
2.7 \\
7.3 \\
40.0 \\
46.4 \\
\end{array}$ & 4.5 & $0.35^{\mathrm{ns}}$ \\
\hline $\begin{array}{l}\text { Occupation of student's } \\
\text { mother. } \\
\text { Working } \\
\text { Unemployed }\end{array}$ & $\begin{array}{l}15 \\
38 \\
\end{array}$ & $\begin{array}{l}28.3 \\
71.7\end{array}$ & $\begin{array}{l}23 \\
34 \\
\end{array}$ & $\begin{array}{l}40.4 \\
59.6 \\
\end{array}$ & $\begin{array}{l}38 \\
72 \\
\end{array}$ & $\begin{array}{l}34.5 \\
65.5 \\
\end{array}$ & $X_{2}=1.76$ & $0.18^{\mathrm{ns}}$ \\
\hline $\begin{array}{l}\text { Occupation of students' } \\
\text { father. } \\
\text { Working } \\
\text { Unemployed }\end{array}$ & $\begin{array}{c}53 \\
0\end{array}$ & $\begin{array}{l}100 \\
0.0\end{array}$ & $\begin{array}{c}54 \\
3 \\
\end{array}$ & $\begin{array}{c}94.7 \\
5.3\end{array}$ & $\begin{array}{c}3 \\
107\end{array}$ & $\begin{array}{c}97.3 \\
2.7\end{array}$ & 1.23 & $0.24^{\mathrm{ns}}$ \\
\hline $\begin{array}{l}\text { Family income. } \\
\text { Just enough } \\
\text { More than enough }\end{array}$ & $\begin{array}{l}29 \\
24 \\
\end{array}$ & $\begin{array}{l}54.7 \\
45.3\end{array}$ & $\begin{array}{l}30 \\
27\end{array}$ & $\begin{array}{l}52.6 \\
47.4 \\
\end{array}$ & $\begin{array}{l}59 \\
51\end{array}$ & $\begin{array}{l}53.6 \\
46.4 \\
\end{array}$ & $\mathrm{X}_{2}=0.05$ & $0.83^{\mathrm{ns}}$ \\
\hline $\begin{array}{l}\text { Weight kg. } \\
\text { Average } \\
\text { Mean } \pm \text { SD }\end{array}$ & \multicolumn{2}{|c|}{$\begin{array}{c}46 \text { to } 82 \\
57.92 \pm 8.97\end{array}$} & \multicolumn{2}{|c|}{$\begin{array}{c}45 \text { to } 89 \\
59.19 \pm 10.2\end{array}$} & \multicolumn{2}{|c|}{$\begin{array}{c}45 \text { to } 89 \\
58.58 \pm 9.6 \\
\end{array}$} & $\mathrm{t}=0.69$ & $0.49^{\mathrm{ns}}$ \\
\hline $\begin{array}{l}\text { Height } \mathrm{cm} . \\
\text { Average } \\
\text { Mean } \pm \text { SD }\end{array}$ & \multicolumn{2}{|c|}{$\begin{array}{c}145 \text { to } 169 \\
160.24 \pm 7.11\end{array}$} & \multicolumn{2}{|c|}{$\begin{array}{l}152 \text { to } 169 \\
160.51 \pm 4.9\end{array}$} & \multicolumn{2}{|c|}{$\begin{array}{c}145 \text { to } 169 \\
160.38 \pm 6.04\end{array}$} & $\mathrm{t}=0.24$ & $0.81^{\mathrm{ns}}$ \\
\hline $\begin{array}{l}\left.\text { BMI (kg. } / \mathbf{m}^{2}{ }^{2}\right) \\
\text { Under-weight }(<18.50 \\
\mathrm{kg} . / \mathrm{m} .2) \\
\text { Normal } \quad(18.50-24.9 \\
\mathrm{kg} . / \mathrm{m} .2) \\
\text { Overweight }(25.00-29.9 \\
\mathrm{kg} . / \mathrm{m} .2) \\
\text { Obese }(\geq 30 \mathrm{~kg} . / \mathrm{m} .2)\end{array}$ & $\begin{array}{l}2 \\
37 \\
13 \\
1\end{array}$ & $\begin{array}{c}3.8 \\
69.8 \\
24.5 \\
1.9\end{array}$ & $\begin{array}{l}3 \\
42 \\
9 \\
3\end{array}$ & $\begin{array}{c}5.3 \\
73.7 \\
15.8 \\
5.3\end{array}$ & $\begin{array}{c}5 \\
79 \\
22 \\
4\end{array}$ & $\begin{array}{c}4.5 \\
71.8 \\
20.0 \\
3.6\end{array}$ & $\begin{array}{c}2.1 \\
t=0.64\end{array}$ & $\begin{array}{l}0.56^{\mathrm{ns}} \\
0.52^{\mathrm{ns}}\end{array}$ \\
\hline Mean \pm SD. & \multicolumn{2}{|c|}{$22.53 \pm 2.92$} & \multicolumn{2}{|c|}{$22.92 \pm 3.41$} & \multicolumn{2}{|c|}{$22.73 \pm 3.17$} & $\mathrm{t}=0.64$ & $0.52^{\mathrm{ns}}$ \\
\hline
\end{tabular}

FET: Fisher exact test.

$\mathrm{X}^{2}$ : chi square test.

${ }^{\text {ns }} \mathbf{P}>\mathbf{0 . 0 5}$.

Table (2): Distribution of the studied sample according to menstrual history before nursing intervention: $(\mathrm{N}=110)$. 
Comparison of Effectiveness of Fennel versus Vitamin (E) on Primary Dysmenorrhea among Benha University Nursing Students

\begin{tabular}{|c|c|c|c|c|c|c|c|c|}
\hline \multirow[t]{2}{*}{ Menstrual History } & \multicolumn{2}{|c|}{$\begin{array}{c}\text { Fennel Group } 1 \\
\quad(\mathrm{~N}=53)\end{array}$} & \multicolumn{2}{|c|}{$\begin{array}{c}\text { Vitamin } E \\
\text { Group } 2(N=57)\end{array}$} & \multicolumn{2}{|c|}{ Total } & \multirow[t]{2}{*}{ FET } & \multirow[t]{2}{*}{ P-Value } \\
\hline & No. & $\%$. & No. & $\%$. & No. & $\%$. & & \\
\hline $\begin{array}{l}\text { Age at menarche. } \\
\text { (9-11) years old } \\
(12-15) \text { years old } \\
(\geq 16) \text { years old }\end{array}$ & $\begin{array}{c}3 \\
36 \\
14\end{array}$ & $\begin{array}{r}5.7 \\
67.9 \\
26.4\end{array}$ & $\begin{array}{c}3 \\
38 \\
16\end{array}$ & $\begin{array}{c}5.3 \\
66.7 \\
28.1\end{array}$ & $\begin{array}{c}6 \\
74 \\
30\end{array}$ & $\begin{array}{c}5.5 \\
67.3 \\
27.3\end{array}$ & 0.14 & $1.0^{\mathrm{ns}}$ \\
\hline Mean \pm SD. & \multicolumn{2}{|c|}{$13.25 \pm 3.23$} & \multicolumn{2}{|c|}{$14.01 \pm 2.47$} & \multicolumn{2}{|c|}{$\frac{1}{13.76 \pm 1.98}$} & $\mathrm{t}=1.38$ & $0.08^{\mathrm{ns}}$ \\
\hline $\begin{array}{l}\text { Menstrual duration. } \\
\text { Average } \\
\text { Mean } \pm \mathrm{SD}\end{array}$ & \multicolumn{2}{|c|}{$\begin{array}{c}3 \text { to } 8 \\
5.72 \pm 1.18\end{array}$} & \multicolumn{2}{|c|}{$\begin{array}{c}3 \text { to } 12 \\
6.0 \pm 1.78\end{array}$} & \multicolumn{2}{|c|}{$\begin{array}{c}3 \text { to } 12 \\
5.86 \pm 1.52\end{array}$} & $\mathrm{t}=0.97$ & $0.33^{\mathrm{ns}}$ \\
\hline $\begin{array}{l}\text { Menstrual interval. } \\
\text { Average } \\
\text { Mean } \pm \text { SD }\end{array}$ & \multicolumn{2}{|c|}{$\begin{array}{c}21 \text { to } 32 \\
27.32 \pm 3.71\end{array}$} & \multicolumn{2}{|c|}{$\begin{array}{c}21 \text { to } 32 \\
27.35 \pm 3.7\end{array}$} & \multicolumn{2}{|c|}{$\begin{array}{c}21 \text { to } 32 \\
27.34 \pm 3.69\end{array}$} & $\mathrm{t}=0.043$ & $0.97^{\mathrm{ns}}$ \\
\hline $\begin{array}{l}\text { Information about menses. } \\
\text { Yes } \\
\text { No }\end{array}$ & $\begin{array}{l}37 \\
16\end{array}$ & $\begin{array}{l}69.8 \\
30.2\end{array}$ & $\begin{array}{l}47 \\
10\end{array}$ & $\begin{array}{l}82.5 \\
17.5\end{array}$ & $\begin{array}{l}84 \\
26\end{array}$ & $\begin{array}{l}76.4 \\
23.6\end{array}$ & $X^{2}=2.43$ & $0.12^{\mathrm{ns}}$ \\
\hline $\begin{array}{l}\text { Source of menstrual } \\
\text { information. } \\
\text { Mother } \\
\text { Sister } \\
\text { Friends } \\
\text { Others } \\
\end{array}$ & $\begin{array}{c}29 \\
1 \\
3 \\
17\end{array}$ & $\begin{array}{c}54.7 \\
1.9 \\
5.7 \\
32.1\end{array}$ & $\begin{array}{c}32 \\
6 \\
0 \\
14\end{array}$ & $\begin{array}{c}56.1 \\
10.5 \\
0.0 \\
24.6\end{array}$ & $\begin{array}{c}61 \\
7 \\
3 \\
31\end{array}$ & $\begin{array}{c}55.5 \\
6.4 \\
2.7 \\
28.2\end{array}$ & $\begin{array}{c}X^{2}=0.023 \\
\text { FET }=2.14 \\
\text { FET }=1.53 \\
X^{2}=0.77\end{array}$ & $\begin{array}{l}0.88^{\mathrm{ns}} \\
0.12^{\mathrm{ns}} \\
0.11^{\mathrm{ns}} \\
0.38^{\mathrm{ns}}\end{array}$ \\
\hline
\end{tabular}

FET: Fisher exact test.

t test: Student's t-test.

$\mathrm{X}^{2}$-test: Chi square test.

${ }^{\mathrm{ns}} \mathbf{P}>\mathbf{0 . 0 5}$.

Table (3): Distribution of the studied sample according to menstrual characteristics before nursing intervention: $(\mathrm{N}=110)$.

\begin{tabular}{|c|c|c|c|c|c|c|}
\hline \multirow[t]{2}{*}{ Menstrual Characteristics } & \multicolumn{2}{|c|}{$\begin{array}{c}\text { Fennel Group } 1 \\
(\mathbf{n}=53)\end{array}$} & \multicolumn{2}{|c|}{$\begin{array}{c}\text { Vitamin E } \\
\text { Group } 2(n=57)\end{array}$} & \multirow[t]{2}{*}{$\mathbf{X}^{2}$} & \multirow[t]{2}{*}{ P-Value } \\
\hline & No. & $\%$. & No. & $\%$. & & \\
\hline $\begin{array}{l}\text { Menstrual pain pattern. } \\
\text { Mild pain } \\
\text { Moderate pain } \\
\text { Severe pain } \\
\text { Intolerable pain }\end{array}$ & $\begin{array}{c}3 \\
27 \\
18 \\
5\end{array}$ & $\begin{array}{c}5.7 \\
50.9 \\
34.0 \\
9.4\end{array}$ & $\begin{array}{c}3 \\
22 \\
22 \\
10\end{array}$ & $\begin{array}{c}5.3 \\
38.6 \\
38.6 \\
17.5\end{array}$ & $\mathrm{FET}=2.47$ & $0.51^{\mathrm{ns}}$ \\
\hline $\begin{array}{l}\text { Menstrual pain duration. } \\
\text { Average } \\
\text { Mean } \pm \text { SD } \\
\end{array}$ & \multicolumn{2}{|c|}{$\begin{array}{l}2 \text { to } 5 \\
3.15 \pm 1.01 \\
\end{array}$} & \multicolumn{2}{|c|}{$\begin{array}{l}2 \text { to } 5 \\
2.82 \pm 1.05\end{array}$} & $\mathrm{t}=1.66$ & $0.10^{\mathrm{ns}}$ \\
\hline $\begin{array}{l}\text { Menstrual pain effect on daily } \\
\text { activities. } \\
\text { Yes } \\
\text { No }\end{array}$ & $\begin{array}{c}47 \\
6\end{array}$ & $\begin{array}{l}88.7 \\
11.3\end{array}$ & $\begin{array}{l}45 \\
12\end{array}$ & $\begin{array}{l}78.9 \\
21.1\end{array}$ & 1.9 & $0.17^{\mathrm{ns}}$ \\
\hline $\begin{array}{l}\text { Menstrual pain effect on faculty } \\
\text { attendance. } \\
\text { Yes } \\
\text { No }\end{array}$ & $\begin{array}{l}25 \\
28 \\
\end{array}$ & $\begin{array}{l}47.2 \\
52.8\end{array}$ & $\begin{array}{l}32 \\
25\end{array}$ & $\begin{array}{l}56.1 \\
43.9 \\
\end{array}$ & 0.89 & $0.35^{\mathrm{ns}}$ \\
\hline $\begin{array}{l}\text { No. of days abscentism. } \\
\text { No } \\
1 \text { day } \\
2 \text { days } \\
3 \text { days } \\
4 \text { days }\end{array}$ & $\begin{array}{c}24 \\
4 \\
11 \\
11 \\
3\end{array}$ & $\begin{array}{c}45.3 \\
7.5 \\
20.8 \\
20.8 \\
5.7\end{array}$ & $\begin{array}{l}38 \\
4 \\
6 \\
6 \\
3\end{array}$ & $\begin{array}{c}66.7 \\
7.0 \\
10.5 \\
10.5 \\
5.3\end{array}$ & $\mathrm{FET}=6.02$ & $0.19^{\mathrm{ns}}$ \\
\hline Menstrual pain effect on scholar & & & & & & \\
\hline
\end{tabular}


Comparison of Effectiveness of Fennel versus Vitamin (E) on Primary Dysmenorrhea among Benha University Nursing Students

\begin{tabular}{|c|c|c|c|c|c|c|}
\hline $\begin{array}{l}\text { achievement. } \\
\text { Yes } \\
\text { No }\end{array}$ & $\begin{array}{l}27 \\
26 \\
\end{array}$ & $\begin{array}{l}50.9 \\
49.1 \\
\end{array}$ & $\begin{array}{l}36 \\
21 \\
\end{array}$ & $\begin{array}{l}63.2 \\
36.8 \\
\end{array}$ & 2.22 & $0.14^{\mathrm{ns}}$ \\
\hline $\begin{array}{l}\text { Amount of bleeding (pads/day). } \\
\text { Mild ( } \leq 2 \text { pads/day) } \\
\text { Moderate ( } 3-5 \text { pads/day) } \\
\text { Severe ( } \geq 6 \text { pads/day) }\end{array}$ & $\begin{array}{c}5 \\
39 \\
9\end{array}$ & $\begin{array}{c}9.4 \\
73.6 \\
17.0\end{array}$ & $\begin{array}{c}9 \\
40 \\
8\end{array}$ & $\begin{array}{l}15.8 \\
70.2 \\
14.0\end{array}$ & 1.07 & $0.59^{\mathrm{ns}}$ \\
\hline $\begin{array}{l}\text { Pattern of blood. } \\
\text { Liquid } \\
\text { Clotted }\end{array}$ & $\begin{array}{l}31 \\
22 \\
\end{array}$ & $\begin{array}{l}58.5 \\
41.5 \\
\end{array}$ & $\begin{array}{l}36 \\
21 \\
\end{array}$ & $\begin{array}{l}63.2 \\
36.8 \\
\end{array}$ & 0.25 & $0.62^{\mathrm{ns}}$ \\
\hline $\begin{array}{l}\text { Information about Fennel. } \\
\text { Yes } \\
\text { No }\end{array}$ & $\begin{array}{l}13 \\
40 \\
\end{array}$ & $\begin{array}{l}24.5 \\
75.5 \\
\end{array}$ & $\begin{array}{l}12 \\
45 \\
\end{array}$ & $\begin{array}{l}21.1 \\
78.9 \\
\end{array}$ & 0.19 & $0.66^{\mathrm{ns}}$ \\
\hline $\begin{array}{l}\text { Information about Vit E. } \\
\text { Yes } \\
\text { No }\end{array}$ & $\begin{array}{c}7 \\
46\end{array}$ & $\begin{array}{l}13.2 \\
86.8\end{array}$ & $\begin{array}{l}11 \\
46\end{array}$ & $\begin{array}{l}19.3 \\
80.7\end{array}$ & 0.74 & $0.39^{\mathrm{ns}}$ \\
\hline
\end{tabular}

$\mathrm{X}^{2}$-test: Chi square test.

t test: Student's t test.

FET: Fisher exact test.

${ }^{\mathrm{ns}} \mathbf{P}>\mathbf{0 . 0 5}$.

Table (4): Distribution of the studied sample according to menstrual associated symptoms before nursing intervention: $(\mathbf{N}=\mathbf{1 1 0})$.

\begin{tabular}{|c|c|c|c|c|c|c|}
\hline \multirow[t]{2}{*}{ Menstrual Associated Symptoms } & \multicolumn{2}{|c|}{$\begin{array}{c}\text { Fennel Group } 1 \\
(\mathbf{N}=53)\end{array}$} & \multicolumn{2}{|c|}{$\begin{array}{c}\text { Vitamin E } \\
\text { Group } 2(N=57)\end{array}$} & \multirow[t]{2}{*}{$\mathbf{X}^{2}$} & \multirow[t]{2}{*}{ P-Value } \\
\hline & No. & $\%$. & No. & $\%$. & & \\
\hline $\begin{array}{l}\text { Lumbo-sacral backache. } \\
\text { Yes } \\
\text { No } \\
\end{array}$ & $\begin{array}{c}45 \\
8 \\
\end{array}$ & $\begin{array}{l}84.9 \\
15.1 \\
\end{array}$ & $\begin{array}{c}52 \\
5 \\
\end{array}$ & $\begin{array}{l}91.2 \\
8.8 \\
\end{array}$ & 1.05 & $0.31^{\mathrm{ns}}$ \\
\hline $\begin{array}{l}\text { Pain radiating down anterior thigh. } \\
\text { Yes } \\
\text { No }\end{array}$ & $\begin{array}{l}29 \\
24 \\
\end{array}$ & $\begin{array}{l}54.7 \\
45.3 \\
\end{array}$ & $\begin{array}{l}31 \\
26 \\
\end{array}$ & $\begin{array}{l}54.4 \\
45.6 \\
\end{array}$ & 0.001 & $0.97^{\mathrm{ns}}$ \\
\hline $\begin{array}{l}\text { Breast tenderness. } \\
\text { Yes } \\
\text { No }\end{array}$ & $\begin{array}{l}20 \\
33 \\
\end{array}$ & $\begin{array}{l}37.7 \\
62.3 \\
\end{array}$ & $\begin{array}{l}14 \\
43 \\
\end{array}$ & $\begin{array}{l}24.6 \\
75.4\end{array}$ & 2.23 & $0.14^{\mathrm{ns}}$ \\
\hline \begin{tabular}{|l|} 
Nausea. \\
Yes \\
No
\end{tabular} & $\begin{array}{l}19 \\
34\end{array}$ & $\begin{array}{l}35.8 \\
64.2 \\
\end{array}$ & $\begin{array}{l}20 \\
37\end{array}$ & $\begin{array}{l}35.1 \\
64.9\end{array}$ & 0.007 & $0.93^{\mathrm{ns}}$ \\
\hline \begin{tabular}{|l|} 
Vomiting. \\
Yes \\
No \\
\end{tabular} & $\begin{array}{l}15 \\
38 \\
\end{array}$ & $\begin{array}{l}28.3 \\
71.7 \\
\end{array}$ & $\begin{array}{l}17 \\
40 \\
\end{array}$ & $\begin{array}{l}29.8 \\
70.2 \\
\end{array}$ & 0.031 & $0.86^{\mathrm{ns}}$ \\
\hline \begin{tabular}{|l|} 
Diarrhea. \\
Yes \\
No \\
\end{tabular} & $\begin{array}{l}17 \\
36 \\
\end{array}$ & $\begin{array}{l}32.1 \\
67.9 \\
\end{array}$ & $\begin{array}{l}26 \\
31 \\
\end{array}$ & $\begin{array}{l}45.6 \\
54.4\end{array}$ & 2.11 & $0.15^{\mathrm{ns}}$ \\
\hline \begin{tabular}{|l|} 
Headache. \\
Yes \\
No \\
\end{tabular} & $\begin{array}{l}26 \\
27 \\
\end{array}$ & $\begin{array}{l}49.1 \\
50.9 \\
\end{array}$ & $\begin{array}{l}30 \\
27 \\
\end{array}$ & $\begin{array}{l}52.6 \\
47.4\end{array}$ & 0.14 & $0.71^{\mathrm{ns}}$ \\
\hline \begin{tabular}{|l|} 
Dizziness. \\
Yes \\
No \\
\end{tabular} & $\begin{array}{l}31 \\
22 \\
\end{array}$ & $\begin{array}{l}58.5 \\
41.5\end{array}$ & $\begin{array}{l}33 \\
24 \\
\end{array}$ & $\begin{array}{l}57.9 \\
42.1\end{array}$ & 0.004 & $0.95^{\mathrm{ns}}$ \\
\hline \begin{tabular}{|l|} 
Fainting. \\
Yes \\
No \\
\end{tabular} & $\begin{array}{c}6 \\
47 \\
\end{array}$ & $\begin{array}{l}11.3 \\
88.7\end{array}$ & $\begin{array}{c}2 \\
55 \\
\end{array}$ & $\begin{array}{c}3.5 \\
96.5 \\
\end{array}$ & $\mathrm{FET}=1.46$ & $0.15^{\mathrm{ns}}$ \\
\hline $\begin{array}{l}\text { Visual analog score (VAS). } \\
\text { Range } \\
\text { mean } \pm \mathrm{SD}\end{array}$ & & & & & $\mathrm{t}=1.78$ & $0.078^{\mathrm{ns}}$ \\
\hline
\end{tabular}

$\mathrm{X}^{2}$-test: Chi square test.

FET: Fisher exact test. 
Comparison of Effectiveness of Fennel versus Vitamin (E) on Primary Dysmenorrhea among Benha University Nursing Students

t test: Student's t-test.

${ }^{\mathrm{n} s} \mathbf{P}>\mathbf{0 . 0 5}$.

Table (5): Distribution of the studied sample related to menstrual characteristics one month after nursing intervention: $(N=110)$.

\begin{tabular}{|c|c|c|c|c|c|c|}
\hline \multirow[t]{2}{*}{ Menstrual Characteristics } & \multicolumn{2}{|c|}{$\begin{array}{l}\text { Fennel } \\
\text { Group } 1 \\
(\mathrm{~N}=\mathbf{5 3})\end{array}$} & \multicolumn{2}{|c|}{$\begin{array}{l}\text { Vitamin E } \\
\text { Group } 2 \\
(\mathrm{~N}=57)\end{array}$} & \multirow[t]{2}{*}{$\mathbf{X}^{2}$} & \multirow[t]{2}{*}{ P-Value } \\
\hline & No. & $\%$. & No. & $\%$. & & \\
\hline $\begin{array}{l}\text { Menstrual duration. } \\
\text { Average } \\
\text { Mean } \pm \text { SD }\end{array}$ & \multicolumn{2}{|c|}{$\begin{array}{l}3 \text { to } 7 \\
4.92 \pm 0.94\end{array}$} & \multicolumn{2}{|c|}{$\begin{array}{l}3 \text { to } 10 \\
4.67 \pm 1.61\end{array}$} & $\mathrm{t}=1.02$ & $0.31^{\mathrm{ns}}$ \\
\hline $\begin{array}{l}\text { Menstrual interval. } \\
\text { Average } \\
\text { Mean } \pm \text { SD }\end{array}$ & \multicolumn{2}{|c|}{$\begin{array}{l}21 \text { to } 32 \\
26.75 \pm 3.32\end{array}$} & \multicolumn{2}{|c|}{$\begin{array}{l}21 \text { to } 32 \\
26.18 \pm 3.64\end{array}$} & $\mathrm{t}=0.87$ & $0.39^{\mathrm{ns}}$ \\
\hline $\begin{array}{l}\text { Menstrual pain pattern. } \\
\text { No pain } \\
\text { Mild pain } \\
\text { Moderate pain } \\
\text { Severe pain }\end{array}$ & $\begin{array}{l}4 \\
22 \\
19 \\
8\end{array}$ & $\begin{array}{l}7.5 \\
41.5 \\
35.8 \\
15.1\end{array}$ & $\begin{array}{l}3 \\
20 \\
24 \\
10\end{array}$ & $\begin{array}{l}5.3 \\
35.1 \\
42.1 \\
17.5\end{array}$ & $\mathrm{FET}=0.98$ & $0.83^{\mathrm{ns}}$ \\
\hline $\begin{array}{l}\text { Menstrual pain duration (days). } \\
\text { Average } \\
\text { Mean } \pm S D\end{array}$ & \multicolumn{2}{|c|}{$\begin{array}{l}0 \text { to } 3 \\
2.06 \pm 0.59\end{array}$} & \multicolumn{2}{|c|}{$\begin{array}{l}0 \text { to } 3 \\
1.78 \pm 0.63\end{array}$} & $\mathrm{t}=2.34$ & $0.021 *$ \\
\hline $\begin{array}{l}\text { Pain effect on daily activity } \\
\text { Yes } \\
\text { No }\end{array}$ & $\begin{array}{l}38 \\
15\end{array}$ & $\begin{array}{l}71.7 \\
28.3\end{array}$ & $\begin{array}{l}42 \\
15\end{array}$ & $\begin{array}{l}73.7 \\
26.3\end{array}$ & 0.06 & $0.82^{\mathrm{ns}}$ \\
\hline $\begin{array}{l}\text { Pain effect on faculty attendance. } \\
\text { Yes } \\
\text { No }\end{array}$ & $\begin{array}{l}20 \\
33\end{array}$ & $\begin{array}{l}37.7 \\
62.3\end{array}$ & $\begin{array}{l}27 \\
30\end{array}$ & $\begin{array}{l}47.4 \\
52.6\end{array}$ & 1.04 & $0.31^{\mathrm{ns}}$ \\
\hline $\begin{array}{l}\text { No. of days abscentism. } \\
\text { No } \\
1 \text { day } \\
2 \text { days } \\
\end{array}$ & $\begin{array}{l}11 \\
28 \\
14\end{array}$ & $\begin{array}{l}20.8 \\
52.8 \\
26.4\end{array}$ & $\begin{array}{l}8 \\
33 \\
16 \\
\end{array}$ & $\begin{array}{l}14.0 \\
57.9 \\
28.1\end{array}$ & 0.87 & $0.65^{\mathrm{ns}}$ \\
\hline $\begin{array}{l}\text { Pain effect on scholar achievements. } \\
\text { Yes } \\
\text { No }\end{array}$ & $\begin{array}{l}20 \\
33\end{array}$ & $\begin{array}{l}37.7 \\
62.3\end{array}$ & $\begin{array}{l}25 \\
32\end{array}$ & $\begin{array}{l}43.9 \\
56.1\end{array}$ & 0.43 & $0.51^{\mathrm{ns}}$ \\
\hline $\begin{array}{l}\text { Amount of bleeding (pads/day). } \\
\text { Mild ( } \leq 2 \text { pads/day) } \\
\text { Moderate (3-5 pads/day) }\end{array}$ & $\begin{array}{l}16 \\
37\end{array}$ & $\begin{array}{l}30.2 \\
69.8\end{array}$ & $\begin{array}{l}17 \\
40\end{array}$ & $\begin{array}{l}29.8 \\
70.2\end{array}$ & 0.002 & $0.97^{\mathrm{ns}}$ \\
\hline $\begin{array}{l}\text { Pattern of blood. } \\
\text { Liquid } \\
\text { Clotted }\end{array}$ & $\begin{array}{l}35 \\
18\end{array}$ & $\begin{array}{l}66.0 \\
34.0 \\
\end{array}$ & $\begin{array}{l}37 \\
20\end{array}$ & $\begin{array}{l}64.9 \\
35.1 \\
\end{array}$ & 0.02 & $0.90^{\mathrm{ns}}$ \\
\hline
\end{tabular}

X2-test: Chi square test.

ns P>0.05.

$t$ test: Student's t-test.

$* \mathbf{p}<0.05$.

FET: Fisher exact test. 
Table (6): Distribution of the studied sample according to menstrual associated symptoms one month after intervention: $(\mathrm{N}=110)$.

\begin{tabular}{|c|c|c|c|c|c|c|}
\hline \multirow[t]{2}{*}{ Menstrual Associated Symptoms } & \multicolumn{2}{|c|}{$\begin{array}{l}\begin{array}{l}\text { Fennel group } 1 \\
(\mathrm{n}=53)\end{array} \\
\end{array}$} & \multicolumn{2}{|c|}{$\begin{array}{l}\text { Vitamin E group } \\
2(n=57)\end{array}$} & \multirow[t]{2}{*}{$\mathbf{X}^{2}$} & \multirow[t]{2}{*}{ P-Value } \\
\hline & No. & $\%$. & No. & $\%$. & & \\
\hline $\begin{array}{l}\text { Lumbo-sacral backache. } \\
\text { Severe } \\
\text { Moderate } \\
\text { Mild } \\
\text { No }\end{array}$ & $\begin{array}{l}22 \\
10 \\
9 \\
12 \\
\end{array}$ & $\begin{array}{l}41.5 \\
18.9 \\
17.0 \\
22.6\end{array}$ & $\begin{array}{l}25 \\
12 \\
12 \\
8 \\
\end{array}$ & $\begin{array}{l}43.9 \\
21.1 \\
21.1 \\
14.0\end{array}$ & 1.46 & $0.69^{\mathrm{ns}}$ \\
\hline $\begin{array}{l}\text { Pain radiating down anterior thigh. } \\
\text { Severe } \\
\text { Moderate } \\
\text { Mild } \\
\text { No } \\
\end{array}$ & $\begin{array}{l}13 \\
9 \\
3 \\
28 \\
\end{array}$ & $\begin{array}{l}24.5 \\
17.0 \\
5.7 \\
52.8 \\
\end{array}$ & $\begin{array}{l}15 \\
11 \\
2 \\
29 \\
\end{array}$ & $\begin{array}{l}26.3 \\
19.3 \\
3.5 \\
50.9 \\
\end{array}$ & $\mathrm{FET}=0.52$ & $0.96^{\mathrm{ns}}$ \\
\hline $\begin{array}{l}\text { Breast tenderness. } \\
\text { Yes } \\
\text { No }\end{array}$ & $\begin{array}{l}18 \\
35 \\
\end{array}$ & $\begin{array}{l}34.0 \\
66.0 \\
\end{array}$ & $\begin{array}{l}12 \\
45 \\
\end{array}$ & $\begin{array}{l}21.1 \\
78.9 \\
\end{array}$ & 2.31 & $0.13^{\mathrm{ns}}$ \\
\hline $\begin{array}{l}\text { Nausea. } \\
\text { Yes } \\
\text { No }\end{array}$ & $\begin{array}{l}15 \\
38 \\
\end{array}$ & $\begin{array}{l}28.3 \\
71.7 \\
\end{array}$ & $\begin{array}{l}19 \\
38 \\
\end{array}$ & $\begin{array}{l}33.3 \\
66.7 \\
\end{array}$ & 0.33 & $0.57^{\mathrm{ns}}$ \\
\hline $\begin{array}{l}\text { Vomiting } \\
\text { Yes } \\
\text { No }\end{array}$ & $\begin{array}{l}15 \\
38\end{array}$ & $\begin{array}{l}28.3 \\
71.7\end{array}$ & $\begin{array}{l}19 \\
38\end{array}$ & $\begin{array}{l}33.3 \\
66.7\end{array}$ & 0.33 & $0.57^{\mathrm{ns}}$ \\
\hline $\begin{array}{l}\text { Diarrhea. } \\
\text { Yes } \\
\text { No }\end{array}$ & $\begin{array}{l}15 \\
38 \\
\end{array}$ & $\begin{array}{l}28.3 \\
71.7 \\
\end{array}$ & $\begin{array}{l}22 \\
35 \\
\end{array}$ & $\begin{array}{l}38.6 \\
61.4 \\
\end{array}$ & 1.3 & $0.25^{\mathrm{ns}}$ \\
\hline $\begin{array}{l}\text { Headache. } \\
\text { Yes } \\
\text { No }\end{array}$ & $\begin{array}{l}20 \\
33 \\
\end{array}$ & $\begin{array}{l}37.7 \\
62.3 \\
\end{array}$ & $\begin{array}{l}27 \\
30 \\
\end{array}$ & $\begin{array}{l}47.4 \\
52.6\end{array}$ & 1.04 & $0.31^{\mathrm{ns}}$ \\
\hline $\begin{array}{l}\text { Dizziness. } \\
\text { Yes } \\
\text { No }\end{array}$ & $\begin{array}{l}29 \\
24 \\
\end{array}$ & $\begin{array}{l}54.7 \\
45.3 \\
\end{array}$ & $\begin{array}{l}31 \\
26 \\
\end{array}$ & $\begin{array}{l}54.4 \\
45.6 \\
\end{array}$ & 0.001 & $0.97^{\mathrm{ns}}$ \\
\hline $\begin{array}{l}\text { Fainting } \\
\text { Yes } \\
\text { No } \\
\end{array}$ & $\begin{array}{l}5 \\
48 \\
\end{array}$ & $\begin{array}{l}9.4 \\
90.6\end{array}$ & $\begin{array}{l}2 \\
55 \\
\end{array}$ & $\begin{array}{l}3.5 \\
96.5 \\
\end{array}$ & $\mathrm{FET}=0.78$ & $0.26^{\mathrm{ns}}$ \\
\hline $\begin{array}{l}\text { Visual analog Score. } \\
\text { Range } \\
\text { mean } \pm \text { SD }\end{array}$ & \multicolumn{2}{|c|}{$\begin{array}{l}1-8 \\
3.58 \pm 1.87\end{array}$} & \multicolumn{2}{|c|}{$\begin{array}{l}1-8 \\
3.53 \pm 2.18\end{array}$} & $\mathrm{t}=0.15$ & $0.88^{\mathrm{ns}}$ \\
\hline
\end{tabular}

$\mathrm{X}^{2}$-test: Chi square test.

FET: Fisher exact test. t test: Student's t-test.

${ }^{\mathrm{ns}} \mathbf{P}>\mathbf{0 . 0 5}$. 


\section{Comparison of Effectiveness of Fennel versus Vitamin (E) on Primary Dysmenorrhea among Benha University Nursing Students}

Table (7): Distribution of the studied sample related to menstrual characteristics at $3^{\text {rd }}$ month follow-up: $(\mathrm{N}=110)$.

\begin{tabular}{|c|c|c|c|c|c|c|}
\hline \multirow[t]{2}{*}{ Menstrual Characteristics } & \multicolumn{2}{|c|}{$\begin{array}{l}\text { Fennel Group } 1 \\
(\mathbf{N}=53)\end{array}$} & \multicolumn{2}{|c|}{$\begin{array}{l}\text { Vitamin E } \\
\text { Group 2 } \\
(\mathrm{N}=57)\end{array}$} & \multirow{2}{*}{$\mathbf{X}^{2}$} & \multirow{2}{*}{ P-Value } \\
\hline & No. & $\%$ & No. & $\%$ & & \\
\hline $\begin{array}{l}\text { Menstrual duration. } \\
\text { Average } \\
\text { Mean } \pm \text { SD }\end{array}$ & \multicolumn{2}{|c|}{$\begin{array}{l}3 \text { to } 7 \\
4.43 \pm 0.99\end{array}$} & \multicolumn{2}{|c|}{$\begin{array}{l}3 \text { to } 7 \\
4.16 \pm 1.08\end{array}$} & $\mathrm{t}=1.39$ & $0.17^{\mathrm{ns}}$ \\
\hline $\begin{array}{l}\text { Menstrual interval. } \\
\text { Average } \\
\text { Mean } \pm \mathrm{SD}\end{array}$ & \multicolumn{2}{|c|}{$\begin{array}{l}21 \text { to } 30 \\
25.53 \pm 3.23\end{array}$} & \multicolumn{2}{|c|}{$\begin{array}{l}21 \text { to } 28 \\
24.81 \pm 3.06\end{array}$} & $\mathrm{t}=1.2$ & $0.23^{\mathrm{ns}}$ \\
\hline $\begin{array}{l}\text { Menstrual pain pattern. } \\
\text { No pain } \\
\text { Mild pain } \\
\text { Moderate pain }\end{array}$ & $\begin{array}{c}22 \\
30 \\
1\end{array}$ & $\begin{array}{c}41.5 \\
56.6 \\
1.9\end{array}$ & $\begin{array}{l}11 \\
26 \\
20\end{array}$ & $\begin{array}{l}19.3 \\
45.6 \\
35.1\end{array}$ & 21.03 & $0.001 * *$ \\
\hline $\begin{array}{l}\text { Menstrual pain duration. } \\
\text { Average } \\
\text { Mean } \pm \text { SD }\end{array}$ & \multicolumn{2}{|c|}{$\begin{array}{c}0 \text { to } 2 \\
1.34 \pm 0.48\end{array}$} & \multicolumn{2}{|c|}{$\begin{array}{c}0 \text { to } 3 \\
1.28 \pm 0.50\end{array}$} & $\mathrm{t}=0.56$ & $0.58^{\mathrm{ns}}$ \\
\hline $\begin{array}{l}\text { Pain effect on daily activity. } \\
\text { Yes } \\
\text { No }\end{array}$ & $\begin{array}{c}9 \\
44 \\
\end{array}$ & $\begin{array}{l}17.0 \\
83.0 \\
\end{array}$ & $\begin{array}{l}25 \\
32 \\
\end{array}$ & $\begin{array}{l}43.9 \\
56.1 \\
\end{array}$ & 9.29 & $0.002 * *$ \\
\hline $\begin{array}{l}\text { Pain effect on faculty attendance. } \\
\text { Yes } \\
\text { No }\end{array}$ & $\begin{array}{l}10 \\
43 \\
\end{array}$ & $\begin{array}{l}18.9 \\
81.1\end{array}$ & $\begin{array}{l}18 \\
39 \\
\end{array}$ & $\begin{array}{l}31.6 \\
68.4 \\
\end{array}$ & 2.34 & $0.13^{\text {ns }}$ \\
\hline $\begin{array}{l}\text { No. of days abscentism. } \\
\text { No } \\
1 \text { day }\end{array}$ & $\begin{array}{l}36 \\
17\end{array}$ & $\begin{array}{l}67.9 \\
32.1\end{array}$ & $\begin{array}{l}26 \\
31\end{array}$ & $\begin{array}{l}45.6 \\
54.4\end{array}$ & 5.56 & $0.018^{*}$ \\
\hline $\begin{array}{l}\text { Pain effect on scholar achievements. } \\
\text { Yes } \\
\text { No }\end{array}$ & $\begin{array}{l}10 \\
43 \\
\end{array}$ & $\begin{array}{l}18.9 \\
81.1\end{array}$ & $\begin{array}{l}18 \\
39 \\
\end{array}$ & $\begin{array}{l}31.6 \\
68.4 \\
\end{array}$ & 2.34 & $0.13^{\mathrm{ns}}$ \\
\hline $\begin{array}{l}\text { Amount of bleeding (pads/day). } \\
\text { Mild ( } \leq 2 \text { pads/day) } \\
\text { Moderate (3-5 pads/day) }\end{array}$ & $\begin{array}{l}39 \\
14\end{array}$ & $\begin{array}{l}73.6 \\
26.4\end{array}$ & $\begin{array}{l}31 \\
26\end{array}$ & $\begin{array}{l}54.4 \\
45.6\end{array}$ & 4.38 & $0.036^{*}$ \\
\hline $\begin{array}{l}\text { Pattern of blood. } \\
\text { Liquid } \\
\text { Clotted }\end{array}$ & $\begin{array}{c}45 \\
8\end{array}$ & $\begin{array}{l}84.9 \\
15.1\end{array}$ & $\begin{array}{l}43 \\
14\end{array}$ & $\begin{array}{l}75.4 \\
24.6\end{array}$ & 1.54 & $0.22^{\mathrm{ns}}$ \\
\hline $\begin{array}{l}X^{2} \text {-test: Chi square test. } \\
\text { t test: Student's t-test. } \\
\text { ns } P>0.05 \text {. }\end{array}$ & & $\begin{array}{l}* \mathbf{p}<1 \\
* * \mathbf{p}\end{array}$ & 05. & & & \\
\hline
\end{tabular}




\section{Comparison of Effectiveness of Fennel versus Vitamin (E) on Primary Dysmenorrhea among Benha University Nursing Students}

Table (8): Distribution of the studied group according to menstrual associated symptoms at $3^{\text {rd }}$ month follow-up: ( $\mathrm{N}=110)$.

\begin{tabular}{|c|c|c|c|c|c|c|}
\hline \multirow[t]{2}{*}{ Menstrual Associated Symptoms } & \multicolumn{2}{|c|}{$\begin{array}{l}\text { Fennel Group } 1 \\
(\mathrm{~N}=53)\end{array}$} & \multicolumn{2}{|c|}{$\begin{array}{l}\text { Vitamin E Group } \\
2(\mathrm{~N}=57)\end{array}$} & \multirow[t]{2}{*}{$\mathbf{X}^{2}$} & \multirow[t]{2}{*}{ P-Value } \\
\hline & No. & $\%$ & No. & $\%$. & & \\
\hline $\begin{array}{l}\text { Lumbo-sacral backache. } \\
\text { Severe } \\
\text { Moderate } \\
\text { No } \\
\end{array}$ & $\begin{array}{l}13 \\
13 \\
27\end{array}$ & $\begin{array}{l}24.5 \\
24.5 \\
50.9\end{array}$ & $\begin{array}{l}22 \\
19 \\
16\end{array}$ & $\begin{array}{l}38.6 \\
33.3 \\
28.1\end{array}$ & 6.12 & $0.047 *$ \\
\hline $\begin{array}{l}\text { Pain radiating down anterior } \\
\text { thigh. } \\
\text { Severe } \\
\text { Moderate } \\
\text { No } \\
\end{array}$ & $\begin{array}{c}8 \\
4 \\
41 \\
\end{array}$ & $\begin{array}{c}15.1 \\
7.5 \\
77.4\end{array}$ & $\begin{array}{l}10 \\
12 \\
35 \\
\end{array}$ & $\begin{array}{l}17.5 \\
21.1 \\
61.4 \\
\end{array}$ & 4.56 & $0.10^{\mathrm{ns}}$ \\
\hline $\begin{array}{l}\text { Breast tenderness. } \\
\text { Yes } \\
\text { No }\end{array}$ & $\begin{array}{c}3 \\
50\end{array}$ & $\begin{array}{c}5.7 \\
94.3\end{array}$ & $\begin{array}{c}7 \\
50\end{array}$ & $\begin{array}{l}12.3 \\
87.7\end{array}$ & $\mathrm{FET}=0.77$ & $0.32^{\mathrm{ns}}$ \\
\hline $\begin{array}{l}\text { Nausea. } \\
\text { Yes } \\
\text { No } \\
\end{array}$ & $\begin{array}{c}4 \\
49 \\
\end{array}$ & $\begin{array}{c}7.5 \\
92.5 \\
\end{array}$ & $\begin{array}{l}12 \\
45 \\
\end{array}$ & $\begin{array}{l}21.1 \\
78.9 \\
\end{array}$ & 4.03 & $0.045^{*}$ \\
\hline $\begin{array}{l}\text { Vomiting. } \\
\text { Yes } \\
\text { No }\end{array}$ & $\begin{array}{c}3 \\
50 \\
\end{array}$ & $\begin{array}{c}5.7 \\
94.3 \\
\end{array}$ & $\begin{array}{l}12 \\
45 \\
\end{array}$ & $\begin{array}{l}21.1 \\
78.9\end{array}$ & 5.53 & $0.019 *$ \\
\hline $\begin{array}{l}\text { Diarrhea. } \\
\text { Yes } \\
\text { No }\end{array}$ & $\begin{array}{c}3 \\
50\end{array}$ & $\begin{array}{c}5.7 \\
94.3\end{array}$ & $\begin{array}{l}11 \\
46\end{array}$ & $\begin{array}{l}19.3 \\
80.7\end{array}$ & 4.6 & $0.032 *$ \\
\hline $\begin{array}{l}\text { Headache. } \\
\text { Yes } \\
\text { No } \\
\end{array}$ & $\begin{array}{c}6 \\
47 \\
\end{array}$ & $\begin{array}{l}11.3 \\
88.7\end{array}$ & $\begin{array}{l}18 \\
39 \\
\end{array}$ & $\begin{array}{l}31.6 \\
68.4 \\
\end{array}$ & 6.61 & $0.01 *$ \\
\hline $\begin{array}{l}\text { Dizziness. } \\
\text { Yes } \\
\text { No } \\
\end{array}$ & $\begin{array}{c}9 \\
44 \\
\end{array}$ & $\begin{array}{l}17.0 \\
83.0\end{array}$ & $\begin{array}{l}22 \\
35 \\
\end{array}$ & $\begin{array}{l}38.6 \\
61.4 \\
\end{array}$ & 6.34 & $0.012 *$ \\
\hline $\begin{array}{l}\text { Fainting. } \\
\text { Yes } \\
\text { No }\end{array}$ & $\begin{array}{c}0 \\
53\end{array}$ & $\begin{array}{c}0.0 \\
100\end{array}$ & $\begin{array}{c}1 \\
56 \\
\end{array}$ & $\begin{array}{c}1.8 \\
98.2\end{array}$ & $\mathrm{FET}=0.0$ & $1.0^{\mathrm{ns}}$ \\
\hline $\begin{array}{l}\text { Visual analog Score (VAS). } \\
\text { Range } \\
\text { Mean } \pm S D\end{array}$ & \multicolumn{2}{|c|}{$\begin{array}{c}1-5 \\
2.03 \pm 1.21\end{array}$} & \multicolumn{2}{|c|}{$\begin{array}{c}1-5 \\
1.67 \pm 0.91\end{array}$} & $\mathrm{t}=1.78$ & $0.078^{\mathrm{ns}}$ \\
\hline
\end{tabular}

t test: Student's t-test.

\section{DISCUSSION}

Dysmenorrhea is pelvic pain usually described as cramping and felt outside the pelvis in the form of backaches. Dysmenorrhea may be accompanied by some systematic symptoms such breast tenderness, insomnia, nausea, vomiting, diarrhea, headache and dizziness (Mularz, et al., 2017).

The study was a quasi-experimental research which hypothesized that the students who received fennel seeds' tea would experience less severe 


\section{Comparison of Effectiveness of Fennel versus Vitamin (E) on Primary Dysmenorrhea among Benha University Nursing Students}

dysmenorrhea compared to the students who would receive vitamin $\mathrm{E}$.

As regards menstrual pain before nursing intervention, less than one fifth had mild pain, more than one third had moderate pain and severe pain. This result agreed with Ortiz, et al., (2015) who mentioned that dysmenorrhea was more in degree of moderate pain in $43.8 \%$ and disagreed in mild pain in $36.1 \%$ and severe pain cases in $20.1 \%$.

Regarding the mean menstrual pain duration, the current study showed that $3.15 \pm 1.01$ for fennel group and 2.82 \pm 1.05 for vitamin $E$ groups at younger age, This result was inconsistent with Tavallaee, (2009) who mentioned that women who experienced pain all the time during their periods, $81.1 \%$ were 30 years old or younger while women who had pain episodes of two hours or more, $74.3 \%$ were below the age of 30 years.

As regard the effect of menstrual pain on daily activity, the majority of the studied sample had menstrual pain, this result agreed with Abdul-Razzak, (2010) who said that more than half of participants $60.4 \%$ reported that dysmenorrhea affected their daily activity for several days each month. This result disagreed with Abd El-Hameed, et al., (2011) who discovered that $66.2 \%$ perform physical activities during menstrual period.

Regarding the effect of menstrual pain on faculty attendance, about half of sample participants were absent due to menstrual pain the number of abscentism days ranged from 1-4 days. This result agreed with Esen, et al., (2016) who reported that the majority of the adolescent girls perceived their periods to be normal. However, mild to moderate dysmenorrhea could have an impact on school attendance and social life.

Regarding the amount of monthly bleeding, more than two thirds of studied sample had moderate amount of monthly bleeding and changed 3-5 pads/day. This result disagreed with Shehata, (2015) who mentioned that $57.0 \%$ of participants had normal amount of blood loss 3-5 pads/day.

The present study revealed that less than one quarter of participants had severe bleeding, This result was consistent with Shehata, (2015) who reported that $8.0 \%$ had heavy blood loss. Also the present study demonstrated that more than half of the studied sample had liquid nature of menstrual blood, and less than half of participants had blood clots in menstrual flow. This result agreed with Shehata, (2015) who mentioned that $66.0 \%$ had liquid blood and $18.0 \%$ had blood clots.

Concerning the information about fennel and vitamin $\mathrm{E}$, more than two thirds of nursing students who received fennel or vitamin $\mathrm{E}$ had no knowledge about fennel and vitamin $\mathrm{E}$ as complementary and alternative methods used in the treatment of dysmenorrhea. This result agreed with Topuz, et al., (2015) who reported that more than half $(58.8 \%)$ of cases believed that their knowledge was insufficient about complementary and alternative medicine (CAM). Also this result disagreed with Jaiswal, et al., (2015) who mentioned that $79.0 \%$ respondents were aware of CAM.

As regards menstrual associated symptoms, the majority of the current studied sample were suffering from lumbo-sacral backache. This result agreed with Al-Jefout (2016) who demonstrated that $62 \%$ of young adult Jordanian females were suffering from lower back pains. This result was incongruent with Kural, et al., (2015) who showed that dysmenorrhea was largely associated with abdominal pain, $34.0 \%$ had pain diffused in lower abdomen followed with suprapubic $22.8 \%$, lower back $16.0 \%$ and thighs $3.4 \%$. Besides $24.0 \%$ of the girls reported that they had pain in all locations.

Related to pain radiating down anterior thigh, the present study concluded that more than half of studied sample were suffering from pain radiating down anterior thigh. This result agreed with 


\section{Comparison of Effectiveness of Fennel versus Vitamin (E) on Primary Dysmenorrhea among Benha University Nursing Students}

Ortiz, et al., (2015) who clarified that near half of dysmenorrheic women $43.1 \%$ suffer from backache. This result disagreed with Bahrami, et al., (2017) who mentioned that leg pain was reported in $18.4 \%$ of adolescents.

As regards breast symptoms, the present study demonstrated that less than half of the students were suffering from breast tenderness. This result was consistent with Ortiz, et al., (2015) who said that $45.4 \%$ of cases had painful or tender breasts.

Concerning the effects of dysmenorrhea on gastro-intestinal tract symptoms, the present study revealed that more than one third students who received either fennel or vitamin $\mathrm{E}$ were suffering from nausea. This result agreed with Sangestani, et al., (2015) who mentioned that $61.8 \%$ of study group and $65.6 \%$ of control group were nauseated. This result wasn't in the same line with Bahrami, et al., (2017) clarified that $4.3 \%$ of the sample were nauseated.

Regarding vomiting, the present study showed that more than one quarter of participants had vomiting. This result agreed with Shaji, (2014) who showed that vomiting was reported in $32 \%$. This result disagreed with Bahrami, et al., (2017) indicated that $4.2 \%$ had vomiting, Also Sangestani et al., (2015) reported that vomiting was common between 17.6 $\%$ the experimental group and $6.2 \%$ in the control group.

Concerning diarrhea, the present study revealed that less than one third of the studied sample were suffering from diarrhea, This result was congruent with Sangestani et al., (2015) who said that diarrhea was common between in $26.5 \%$ in the experimental group and $28.1 \%$ the control group. This result was inconsistent Aboushady and El-saidy, (2016) demonstrated that $7.5 \%$ had diarrhea.

As regards headache, the present study revealed that about half the sample in two groups complained of headache. This result was congruent with Mohamed and Mansour, (2013) who demonstrated that $46.7 \%$ had headache. This result was incongruent with Shaji, (2014) who demonstrated that $29 \%$ had headache.

Concerning complaint of dizziness, more than one third of participants had dizziness. This result agreed with Mohamed and Mansour, (2013) who said that $44.3 \%$ had dizziness. As regard fainting, the present study showed that less than one sixth of participants complained of fainting. This result agreed with Rakhshaee, (2010) who said that $4.0 \%$ suffered from fainting.

The current study showed that after three months of follow-up with fennel tea, the students who had dysmenorrhea experienced fewer menstrual associated symptoms, this is evidenced by significant improvement in menstrual characteristics such as less severe menstrual bleeding amount and pattern, in addition, this led to better daily activity, more faculty attendance and better scholar achievements.

Also this improvement had positive effects on menstrual associated symptoms such as reduced lumbo-sacral pain, less severe pain radiating down anterior thigh, less breast tenderness, improved nausea and vomiting, This recovery might be due to the mechanism of action of fennel through the inhibition of uterine contractions induced by prostaglandin E2 and oxytocin (Kauphal, 2018).

This result was congruent with Khodakarami, et al., (2009) who reported that the intensity of pain decreased from $5.3 \%$ to $3.0 \%$ in the second month and $0.5 \%$ in the third month after fennel. Also Omidvar, et al., (2012) demonstrated that the use of fennel for two months showed more effective results than placebo in pain relief, In addition, Ghodsi and Asltoghiri, (2014) reported that fennel intake reduced pain, menstrual bleeding, nausea and weakness after three month periods. 


\section{Comparison of Effectiveness of Fennel versus Vitamin (E) on Primary Dysmenorrhea among Benha University Nursing Students}

The present work showed that after three months follow-up with vitamin $\mathrm{E}$, it was found that there were more fewer dysmenorrhea symptoms than before intervention and on month after, This is evidenced by significant improvement in menstrual characteristics, such as reduced menstrual duration, less severe pattern of menstrual pain, fewer menstrual pain duration, less severe menstrual bleeding amount and pattern.

Reduced dysmenorrhea symptoms improved their daily activity, reduced abscentism from the faculty and improved scholar achievements, Also this was associated with less severe lumbo-sacral pain, less severe pain radiating down anterior thigh as well as reduced manifestation of nausea, vomiting, diarrhea, headache and dizziness. This recovery might be due to the mechanism of action of vitamin $E$ acting as antiinflammatory which affects betaendorphin levels (Kauphal, 2018).

This result was consistent with Nasehi, et al., (2013) who mentioned that after utilization of fennel/vitamin $\mathrm{E}$ for two months reduced dysmenorrhea.

Besides Nayeban, et al., (2014) compared the effects of vitamin $\mathrm{E}$ and vitamin B1 on the severity and duration of pain in primary dysmenorrhea and found that vitamin $\mathrm{E}$ could reduce pain duration in primary dysmenorrhea.

Then Atallahi, et al., (2014) reported that the use of wheat germ extract which is rich vitamin $\mathrm{E}$ for two months was an effective treatment for dysmenorrhea and it's systemic symptoms, probably because of its anti-inflammatory effects. Liu and Jacobs, (2017) demonstrated that vitamin E can reduce mastalgia.

The present work showed that at 3rd month follow-up, the group who received fennel tea experienced less severe dysmenorrhea compared to the students who received vitamin $\mathrm{E}$, this evidenced by reducing menstrual characteristics and menstrual associated symptoms like menstrual pain pattern, mean menstrual pain duration, effect of pain on daily activity, and number of days abscentism from faculty, menstrual bleeding, lumbosacral backache, nausea, vomiting, diarrhea, headache and dizziness.

Also this result agreed with Moslemi, et al, (2013) who illustrated that fennel extract proved more effective in pain relief than vitamin E. also Amini, et al., (2016) who reported that fennel seems to be superior to placebo or vitamin $\mathrm{E}$.

\section{CONCLUSION}

Fennel tea and vitamin $\mathrm{E}$ had strong positive effect on dysmenorrhea relief. But students who received fennel seed's tea experienced less severe dysmenorrhea than students who received vitamin $\mathrm{E}$.

\section{RECOMMENDATIONS}

Nurse should encourage females with dysmenorrhea to try Fennel tea or vitamin E for four subsequent menstrual cycles to ensure their efficacy on dysmenorrhea relief (starting from two days before menstrual bleeding onset to three days after menstrual bleeding onset).

\section{Recommendations for further researches:}

This research should be compared with others using combination of both fennel and vitamin $\mathrm{E}$.

\section{REFRENCES}

Abd El-Hameed, A.N., Mohamed, S.M., Ahmed, H.N., and Ahmed, R.E., (2011). Assessment of Dysmenorrhea and Menstrual Hygiene Practices among Adolescent Girls in Some Nursing Schools at EL-Minia Governorate. Egypt, Journal of American Science, 7, PP: (1-10). Retrieved from

http://www.americanscience.org. Accessed on 15-7-2017.

Abdul-Razzak, K.K., Ayoub, M.N., AbuTaleb, A.A., and Obeidat, A.B., (2010). Influence of Dietary Intake 
of Dairy Products on

Dysmenorrhea, J. Obstet. Gynaecol. Res., 36(2), PP: 377-383.

Abo Elnor, R.S., (2017). Effect of Caffeinated Beverages Intake on Premenstrual Syndrome among Nursing Students, master thesis, Faculty of Nursing, Benha University, pp: 96-100.

Abo Elnor, R.S., (2018). Effect of Caffeinated Beverages Intake on Premenstrual Syndrome among Nursing Students, Egyptian Journal of Health Care, 9(3), pp: 1-10.

Abou shady, N.M.R., \& El-saidy, K.M.T., (2016).Effect of Home based Stretching Exercises and Menstrual Care on Primary Dysmenorrhea and Premenstrual Symptoms among Adolescent, IOSR, Journal of Nursing and Health Science, 5(2), pp: 10-17.

Akhlaghi, F., Zirak, N., and Nazemian, S.H. (2009). The effect vitamin E on primary Dysmenorrhea, J. Hayat, 15(1), pp: 13-19.

Al-Jefout, M., Seham, A., Jameel, H., and Randa, A., (2015). Dysmenorrhea: Prevalence and Impact on Quality of Life among Young Adult Jordanian Females, J. Pediatr. Adolesc. Gynecol, 28(3), PP: 173185.

Amini, F., Marzban, M., and Salehi, A., (2016). The Effect of Foeniculum Vulgare on Dysmenorrhea: A Systematic Review, European Journal of Integrative Medicine, (2016), 8(1), pp: 60-61.

Arafa, E.A., Khamis, Y., Hassan, E.H., Saber, M.N., and Abbas, M.A., (2018) Epidemiology of Dysmenorrhea among Workers in Upper Egypt; A Cross Sectional Study, Middle East Fertility Society Journal, 23(1), PP: 44-47.

Atallahi, A., Akbari, A.A., Mojab, F., and Majd, A.H., (2014). Effects of Wheat Germ Extract on the Severity and Systemic Symptoms of
Primary Dysmenorrhea: A Randomized Controlled Clinical Trial, Iran Red Crescent Med. J., 16(8), P: e19503.

Bahrami, A., Sadeghnia, H., Avan, A., Mirmousavi, J.S., and Moslem, A., (2017). Neuropsychological Function in relation to Dysmenorrhea in Adolescents, European Journal of Obstetrics \& Gynecology and Reproductive Biology, 215, PP: 224-229. Retrieved from https.www.clinicalkey.com.mplbci. ekb.eg/\#!/content/journal/1-s2.0$\underline{\text { S030121151730307X? scrollTo }=\% 2}$ 3. Accessed on 30-7-217.

Burnett, M., \& Lemyre, M., (2017). 345Primary Dysmenorrhea Consensus Guideline, J. Obstet. Gynecol. Can, 39(7), PP: 585-595.

Esen, I., Oğuz, B., and Serin, M.H., (2016). Menstrual Characteristics of Pubertal Girls: A QuestionnaireBased Study in Turkey, J Clin Res Pediatr Endocrinol, 8(2), pp: 192196.

Geller, G., \& Simone, R., (2011). Herbs and Supplements (Fennel). EBSCO Complementary and Alternative Medicine (CAM) Review Board. EPSCO Publishing, Retrieved from http://therapy.epnet.com/nat/GetCo ntent.asp?chunkiid $=108303$.

Accessed on 5-7-2017.

Ghodsi, Z., \& Asltoghiri, M., (2014). The Effect of Fennel on Pain Quality, Symptoms, and Menstrual Duration in Primary Dysmenorrhea, Journal of North American Society for Pediatric and Adolescent Gynecology, 27(5), PP: 283-286.

Grunberg, S.M., Groshen, S., Steingass, S., Zaretsky, S., Meyerowitz, B., (1996). Comparison of conditional quality of life terminology and visual analogue scale measurements, Quality of Life Research, 5(1), PP: 65-72. 


\section{Comparison of Effectiveness of Fennel versus Vitamin (E) on Primary Dysmenorrhea among Benha University Nursing Students}

Jaiswal, K., Bajait, C., Pimpalkhute, S., Sontakke, S., Dakhale, G., and Magdum, A., (2015). Knowledge Attitude and Practice of Complementary and Alternative Medicine: A Patient's Perspective, International Journal of Medicine and Public Health, 5(1), P: 19.

Kannan, P., Chapple, C.M., Miller, M., and Claydon, S.L., (2015). Menstrual Pain and Quality of Life in Women with Primary Dysmenorrhea: Rationale Design and Interventions of A Randomized Controlled Trial of Effects of A Treadmill-based Exercise Intervention, Contemporary Clinical Trials, 42(2), PP: 81-89.

Kauphal, J.G., (2018). Integrative Medicine, Chapter 56: Dysmenorrhea, 4th ed, Elsevier Inc. United States. pp: 560-577.

Khodakarami, N., Moatar, F., Ghahari, A., and Soloki, S., (2009). The Effect of Iranian Herbal Drug on Primary Dysmenorrhea: A clinical Controlled Trial, J. Midwifery Women Health, 54(5), pp: 401-404.

Kural, R.M., Noor, N.N., Pandit, D., and Jushi, T., (2015). Menstrual Characteristics and Prevalence of Dysmenorrhea in College Going Girls, J. Family Med Prim Care, (2015), 4(3), PP: 426-4.

Leifer, G., (2015). Introduction to Maternity and Pediatric Nursing. Chapter11: Maternal-Newborn Nursing and Women's Health, 6th ed. Elsevier Sunders, United States of America, p: 254.

Liu, J., \& Jacobs, K.L., (2017). Current Surgical Therapy, the Management of Benign Breast Disease, 20th ed, pp: 657-659.

Mohammed, H., \& Mansour, S., (2013). The Effect of Dysmenorrhea on Quality of Life of Technical Secondary Schools Girls., Med. J. Cairo Univ., 81(2), PP: 83-90.
Moslemi, L., Bekhradi, R., Galini Moghadam, T., and Tabari, M., (2012). Comparative Effect of Fennel Extract on the Intensity of Primary Dysmenorrhea, African Journal of Pharmacy and Pharmacology, 6(24), pp: 17701773.

Mularz, A., Dalati, S., and Pedigo, R., (2017). OB/GYN SECRETS, Premenstrual Syndrome and Dysmenorrhea, 4th ed, Elsevier Inc, United State of America, pp:11-12.

Nasehi, M. Sehhatie, F., Zamanzadeh, V. and Delazar, A., (2013). Comparison of the Effectiveness of Combination of Fennel Extract/Vitamin E with Ibuprofen on the Pain Intensity in Students with Primary Dysmenorrhea, Iran J Nurs., Midwifery Res., 18(5), pp: 9,335 .

Nayeban, S., Jafarnejad, F., Nayeban, S., and Sefidgaran, A., (2014). A Comparison of the Effects of Vitamin E and Vitamin B1 on the Severity and Duration of Pain in Primary Dysmenorrhea., Journal of Midwifery and Reproductive Health., 2(2), pp:143-146.

Omidvar, S., Esmailzadeh, S., Baradaran, M., and Basirat, Z., (2012). Effect of Fennel on Pain Intensity in Dysmenorrhea: A PlaceboControlled Trial, Ayu, 33(2), pp: 311,313.

Ortiz, M., Márquez, S., Quezada, L., and Cánovas, G., (2015). Effect of a Physiotherapy Program in Women with Primary Dysmenorrhea, European journal of Obstetrics \& Gynecology and Reproductive Biology, 194, pp: 24-29.

Osman, H. \& El-Houfey, A., (2016). Prevalence of dysmenorrhea and its impact on quality of life among nursing students at Assiut University Egypt, IOSR-JNHS, 5(1), pp: 8-16. 


\section{Comparison of Effectiveness of Fennel versus Vitamin (E) on Primary Dysmenorrhea among Benha University Nursing Students}

Rakhshaee, Z., (2010). A Cross-sectional Study of Primary Dysmenorrhea among Students at a University: Prevalence, Impact and of Associated Symptoms, Annual Research \& Review in Biology, 4(18), PP: 2815-2822.

Sangestani, G., Khatiban, M., Marci, R., and Piva, I., (2015). The Positive Effects of Zinc Supplements on the Improvement of Primary Dysmenorrhea and Premenstrual Symptoms: A Double-blind, Randomized Controlled Trial, J. Midwifery Reprod. Health, 3(3), PP: 378-384.

Shaji, H., (2014). Severity of Primary Dysmenorrhea and Menstrual Distress among University Students in Kingdom of Saudi Arabia, International Journal of Health Sciences \& Research, 4(11), PP: 209-218.

Shehata, S.N., (2015). Assessment of the Relationship between Body Mass Index and Menstrual Pattern for Female Students, Master Thesis, Faculty of Nursing, Benha University, pp: 60-68.
Sriwatanakul, K. Kelvie, W. Lasagna, L., Calimlim, F.J., (1983). Studies with Different Types of Visual Analog Scales for Measurement of Pain, Clinical Pharmacology, 5(2), pp: 234-239.

Sucato, G., \& Burstein, G., (2016). Nelson Text Book of Pediatrics. Chapter116: Menstrual Problems, 20th ed, Elsevier Inc, pp: 963-969.

Tavallaee, M., (2009). Prevalence of Menstrual Pain and Associated Risk Factors among Iranian Women, Master Thesis. Faculty of Health Science. Simon Fraser University, pp: 35-37.

Topuz, S., Uysal, G., and Yilmaz, A.A., (2015). Knowledge and Opinions of Nursing Students Regarding Complementary and Alternative Medicine for Cancer Patients, International Journal of Caring Sciences, 8(5), P: 656.

Wetwet, A.H. (2016). Effect of Local Heat Application on Intensity of Primary Dysmenorrhea among Nursing Students. Dysmenorrhea, Master Thesis, Faculty of Nursing Benha University, p: 17,24,53,73. 